\title{
Comorbidities and course of lung function in patients with congenital esophageal atresia
}

\author{
Ana Jové Blanco, M.D., ${ }^{a}$ Ana Gutiérrez Vélez, M.D., ${ }^{a}$ Gonzalo Solís-García, M.D., ${ }^{a}$ \\ Antonio Salcedo Posadas, B.S., ${ }^{a}$ Sara Bellón Alonso, B.S., ${ }^{a}$ and \\ Juan Luis Rodríguez Cimadevilla, B.S. ${ }^{a}$
}

\begin{abstract}
Objective. To describe the epidemiological characteristics, hospital follow-up, and course of patients who underwent surgery for esophageal atresia and its consequences on lung function. Population and methods. Retrospective, longitudinal, and analytical study. The medical records of patients with esophageal atresia born between 1996 and 2017 were reviewed. Perinatal data, type of atresia, associated malformations, respiratory and gastrointestinal complications, and spirometry data were recorded over 3 years. Results. A total of 97 patients were included. The most common type of atresia was III, and the most frequent syndrome, trisomy $21 ; 13.4 \%$ of patients died in the neonatal period; $23.8 \%$ were followed up by the Department of Pulmonology, and their respiratory complications included exacerbations $(46.4 \%)$, wheezing or asthma $(36 \%)$, and pneumonia $(26.8 \%)$. Gastroesophageal reflux was a risk factor for wheezing (OR: 5.31; $p=0.002)$, exacerbations(OR:4.00; $p=0.009$ ), and pneumonia (OR:3.24; $p=0.02)$. In the first spirometry $(\mathrm{n}=20)$, the pattern was normal in $65 \%$; restrictive in $30 \%$; and mixed in $5 \%$. In the second spirometry $(\mathrm{n}=19)$, the pattern was normal in $42.1 \%$; restrictive in $31.6 \%$; obstructive in $15.8 \%$, and mixed in $10.5 \%$. In the third spirometry $(\mathrm{n}=14)$, the pattern was normal in $50 \%$; restrictive in $21.4 \%$; obstructive in $14.3 \%$, and mixed in $14.3 \%$. Conclusions. In our sample of patients, a large proportion had respiratory and gastrointestinal comorbidities. Lung function worsened progressively.

Key words: esophageal atresia, lung function tests, children, follow-up studies, comorbidity.
\end{abstract}

Department
of Pediatric

Pulmonology,

Hospital General

Universitario

Gregorio Marañón,

Madrid, Spain.

E-mail address:

Ana Jové Blanco, M.D.: ana.jove@salud.madrid. org

http: / / dx.doi.org/10.5546/ aap.2020.eng.25

To cite: Jové Blanco A, Gutiérrez Vélez A, SolísGarcía G, Salcedo Posadas A, et al. Comorbidities and course of lung function in patients with congenital esophageal atresia. Arch Argent Pediatr 2020;118(1):2530.

\section{Funding: \\ None \\ Conflict of interest: \\ None.}

Received: 2-7-2019

Accepted: 8-1-2019

\section{INTRODUCTION}

Congenital esophageal atresia is a relatively common disease, present in $1 / 2500-4500$ live births. ${ }^{1}$ Thanks to the advances made in the field of neonatal intensive care, the generalization of parenteral nutrition, and the anticipation of surgical treatment, survival of these patients has risen exponentially in recent years. ${ }^{2}$ This has allowed to gain knowledge and study the comorbidities associated with this condition; however, followup of these patients has not become standardized yet.

In approximately half of patients, esophageal atresia is associated with other anatomical malformations (heart, urogenital, gastrointestinal, musculoskeletal or central nervous system malformations). ${ }^{3}$ Sometimes, these malformations are grouped into syndromes/associations, such as VACTERL (vertebral defects, anal atresia, cardiac defects, tracheoesophageal fistula, renal anomalies, and limb abnormalities) or CHARGE (coloboma, heart defects, choanal atresia, retardation of growth, genital abnormalities, and ear abnormalities). Likewise, it is usually present in trisomy 18 (Edwards syndrome) and 21 (Down syndrome), Di George syndrome, and Pierre-Robin syndrome. ${ }^{4}$

Follow-up of these patients is clinical but also functional, based on complementary tests, including forced spirometry. Respiratory complications occur in the short term, as determined by surgery itself, and in the long term, due to respiratory tract and gastrointestinal involvement, which facilitates aspirations or dysphagia. ${ }^{5}$ The studies done so far in the pediatric 
population have demonstrated alterations in lung function tests, with the restrictive pattern as the most common one, although published data on this matter is scarce. ${ }^{1,6}$

For these reasons, the main objective of this study was to describe the epidemiological characteristics, hospital follow-up and course of patients who underwent surgery for esophageal atresia in our hospital, and its consequences on lung function.

\section{MATERIAL AND METHODS}

This was a longitudinal, retrospective, descriptive and analytical study carried out in a national pediatric referral hospital in Spain, leader in Pediatric Neonatology and Surgery. The medical records of patients diagnosed with congenital esophageal atresia born between January 1996 and December 2017 were reviewed.

The study included all patients who underwent surgery for esophageal atresia in our hospital in the study period, regardless of the centre where they had been born, as long as their electronic or paper medical records were available. For the analysis of lung function, patients who died in the neonatal period, those who were lost to follow-up, and those who could not perform a spirometry due to their age or underlying condition were excluded. Perinatal data were recorded, including gestational age, presence of polyhydramnios, lung maturation regimen, and birth weight, as well as chromosomal abnormalities and associated malformations. The type of atresia was recorded based on Ladd's classification ${ }^{7}$ (Table 1), the type of surgery, and time since diagnosis until surgery. In relation to respiratory complications, the number of respiratory exacerbations, pneumonia, recurrent wheezing or asthma events, and the presence of tracheomalacia were determined. The studied gastrointestinal complications included gastroesophageal reflux and aspiration syndrome.

Respiratory exacerbations were defined as events of respiratory worsening diagnosed clinically based on increased cough or respiratory distress, changes in sputum or worsened pulmonary auscultation. Pneumonia was defined only as those events with a radiological confirmation of consolidation. The wheezing/ asthma event category included both acute bronchospasms and respiratory processes that showed wheezing during auscultation. Tracheomalacia, gastro esophageal reflux and aspiration syndrome were assessed in those patients whose follow-up by different specialists showed such events.

Patients under follow-up by the Department of Pediatric Pulmonology of our hospital and the results of 3 spirometries performed during follow-up were recorded. The following spirometry data were assessed: forced expiratory volume in the first second $\left(\mathrm{FEV}_{1}\right)$, forced vital capacity (FVC), and $\mathrm{FEV}_{1} / \mathrm{FVC}$ ratio. The data of the first spirometry done by the patient that met the necessary acceptability and reproducibility standards to consider it valid, and of those performed 1 and 3 years after the first one were recorded. An obstructive pattern was defined as a $\mathrm{FEV}_{1} / \mathrm{FVC}$ below the lower limit of normality (LLN), whereas a restrictive pattern corresponded to a FVC $<80 \%$ of the theoretical value. The information about the treatment required by these patients (aerosol therapy and anti-reflux treatment) was also recorded.

The study was approved by the hospital's Clinical Research Ethics Committee and was conducted in accordance with the guidelines established by the Declaration of Helsinki.

Quantitative outcome measures were described as median and range, and qualitative outcome measures, as percentage. Proportions were compared using the $\chi^{2}$ test or Fisher's exact test and the strength of the association (odds ratio, OR) was established based on a binary logistic regression. Mean values were compared using Student's t test. The statistical significance level was established at $p<0.05$ and the HolmBonferroni correction was used to adjust for

TABLE 1. Ladd's classification. ${ }^{7}$ Types of atresia

\begin{tabular}{ll}
\hline Atresia & Characteristics \\
\hline I & Esophageal atresia with both esophageal pouches ending blindly and without tracheoesophageal fistula. \\
II & Esophageal atresia with upper tracheoesophageal fistula and blind lower esophageal pouch. \\
III & Esophageal atresia with lower tracheoesophageal fistula and blind upper esophageal pouch. \\
IV & Esophageal atresia with tracheoesophageal fistula in both esophageal pouches. \\
V & Tracheoesophageal fistula without esophageal atresia (H-type fistula). \\
\hline
\end{tabular}


multiple comparisons. The statistical analysis was performed with the SPSS software program, version 18.

\section{RESULTS}

A total of 97 patients were included; $57.7 \%$ were males. Their mean birth weight was 2307 grams. The type of atresia and associated malformation syndromes are detailed in Table 1 and Table 2, respectively. Tracheoesophageal fistula was observed in 77 patients $(79.4 \%)$. Perinatal data (gestational age, polyhydramnios, and lung maturation) are summarized in Table 3.

Out of 97 patients included in the study, 13 died in the neonatal period (13.4\%). Mortality was greater among patients with multiple malformations or chromosomal abnormalities $(34.6 \%)$ compared to the rest $(5.7 \%, p<0.001)$. All patients with Edwards syndrome (3), 2 of 8 patients with VACTERL syndrome, and 1 of 10 patients with Down syndrome died. The birth weight of deceased patients was lower than that of survivors (1705 versus $2402 \mathrm{~g}, p=0.002$ ).

The most common access route for surgery was thoracotomy (71 patients, $73.2 \%$ ), followed by thoracoscopy in 6 patients; no significant differences were observed between them in terms of mortality $(p=0.44)$. No data about the type of surgical approach were found in $20.6 \%$ (20) of patients. The median days until the first

TABLE 2. Frequency of types of atresia and associated syndromes

\begin{tabular}{lll}
\hline $\begin{array}{l}\text { Type of atresia } \\
(\mathrm{n}=97)\end{array}$ & I & $18(18.6 \%)$ \\
& II & $1(1 \%)$ \\
& III & $72(74.2 \%)$ \\
& IV & $3(3.1 \%)$ \\
& V & $1(1 \%)$ \\
Associated syndromes & Unknown & $2(2.1 \%)$ \\
$(\mathrm{n}=97)$ & Down syndrome & $9(9.3 \%)$ \\
& VACTERL* & $8(8.2 \%)$ \\
& Edwards syndrome & $3(3.1 \%)$ \\
& Other & $6(6.2 \%)$ \\
& None & $71(72.2 \%)$ \\
\hline
\end{tabular}

VACTERL includes vertebral defects, anal atresia, cardiac defects, tracheoesophageal fistula, renal anomalies, and limb abnormalities.

TABLe 3. Perinatal data

\begin{tabular}{|c|c|c|}
\hline $\begin{array}{l}\text { Polyhydramnios } \\
(\mathrm{n}=38)\end{array}$ & $\begin{array}{l}\text { Total } \\
\text { I } \\
\text { II } \\
\text { III } \\
\text { IV } \\
\text { V }\end{array}$ & $\begin{array}{c}38(39.2 \%) \\
14(77.7 \%) \\
0 \\
20(27.8 \%) \\
0 \\
0\end{array}$ \\
\hline $\begin{array}{l}\text { Gestational age } \\
(\mathrm{n}=97)\end{array}$ & $\begin{array}{c}<28 \text { weeks } \\
29-31 \\
32-36 \\
\text { TNBI }\end{array}$ & $\begin{array}{c}2(2.1 \%) \\
9(9.3 \%) \\
29(29.9 \%) \\
57(58.8 \%)\end{array}$ \\
\hline $\begin{array}{l}\text { Lung maturation } \\
(\mathrm{n}=97)\end{array}$ & $\begin{array}{c}\text { Complete } \\
\text { Incomplete } \\
\text { No } \\
\text { Unknown }\end{array}$ & $\begin{array}{c}14(43.8 \%) \\
3(9.4 \%) \\
5(15.6 \%) \\
10(31.3 \%)\end{array}$ \\
\hline $\begin{array}{l}\text { Birth weight } \\
(\mathrm{n}=97)\end{array}$ & $\begin{array}{c}<1000 \mathrm{~g} \\
1000-2000 \mathrm{~g} \\
2000-3000 \mathrm{~g} \\
3000-4000 \mathrm{~g} \\
>4000 \mathrm{~g}\end{array}$ & $\begin{array}{c}5(5.2 \%) \\
30(30.9 \%) \\
43(44.3 \%) \\
17(17.5 \%) \\
1(1 \%)\end{array}$ \\
\hline
\end{tabular}

TNBI: term newborn infant.

The table shows the percentage of polyhydramnios for each type of atresia. Lung maturation corresponds to newborn infantsborn with $<34$ weeks. 
surgery was 1 (range: 0-163), with a median of hospitalization at the Neonatology unit of 24 days (range: 0-223).

Among the 84 patients that survived, 20 $(23.8 \%)$ were followed up by the Department of Pediatric Pulmonology. Survivors developed the following respiratory complications, by order of frequency: respiratory exacerbations (42 patients, $50 \%$ ), wheezing or asthma events (38 patients, $45.2 \%$ ), pneumonia (26 patients, $30.9 \%$; 5 had a single event and 21, 2 or more), and tracheomalacia (21 patients, $25 \%$ ). In terms of gastrointestinal complications, 56 patients $(66.7 \%)$ had gastroesophageal reflux and 5 $(5.9 \%)$, recurrent aspiration syndrome. Patients with gastroesophageal reflux had a higher risk for wheezing (OR: $5.31 ; p=0.002$ ), respiratory exacerbations (OR: $4.00 ; p=0.009$ ), and pneumonia (OR: $3.24 ; p=0.02$ ).

In relation to treatment, 40 patients $(41.4 \%)$ received maintenance or rescue inhaled drugs. Anti-reflux treatment with ranitidine or proton pump inhibitors was required by 65 patients $(67 \%)$. Treatment for both conditions was required in $30.9 \%$ (30) of patients. Fistula recurrence was observed in 19 patients $(22.6 \%)$.

Among patients with follow-up, the spirometries of 20 patients $(23.8 \%)$ were eligible for assessment. Lung function data could not be obtained in the remaining 64 patients $(76.2 \%)$ because they were not able to perform the spirometry adequately (age, underlying condition, lack of collaboration) or because they had been discharged from the Department of Pulmonology prior to the first spirometry, either because of their favorable course or transfer to a different centre. Lung function data in terms of $\mathrm{FEV}_{1}, \mathrm{FVC}$, and $\mathrm{FEV}_{1} / \mathrm{FVC}$ ratio are shown in Figure 1.

In the first spirometry $(\mathrm{n}=20), 13$ patients $(65 \%)$ had a normal pattern, whereas $6(30 \%)$ showed a restrictive pattern, and $1(5 \%)$, a mixed pattern. Patients who developed pneumonia had a reduced $\mathrm{FEV}_{1}$ in the first spirometry compared to those without pneumonia (79.7 versus 98.9 , $p=0.016)$. In the second spirometry, performed 1 year after the first one $(n=19)$, the spirometry pattern was normal in 8 patients $(42.1 \%)$; restrictive in $6(31.6 \%)$; obstructive in $3(15.8 \%)$; and mixed in $2(10.5 \%)$. In the third spirometry, performed 3 years after the first one $(n=14)$, the spirometry pattern was normal in 7 patients; restrictive in 3; obstructive in 2; and mixed in 2 . According to the third spirometry, again the patients who developed pneumonia had a reduced $\mathrm{FEV}_{1}$, but the results were not statistically significant (72.2 versus $90.2, p=0.053)$.

\section{DISCUSSION}

This study described a cohort of patients who underwent surgery for esophageal atresia at a pediatric referral hospital over the past 20 years. Clinical and epidemiological characteristics at the time of diagnosis, survival, and association with multiple malformation syndromes were similar to those observed in previous studies. ${ }^{5,8}$

FIGURE 1. Lung function parameters

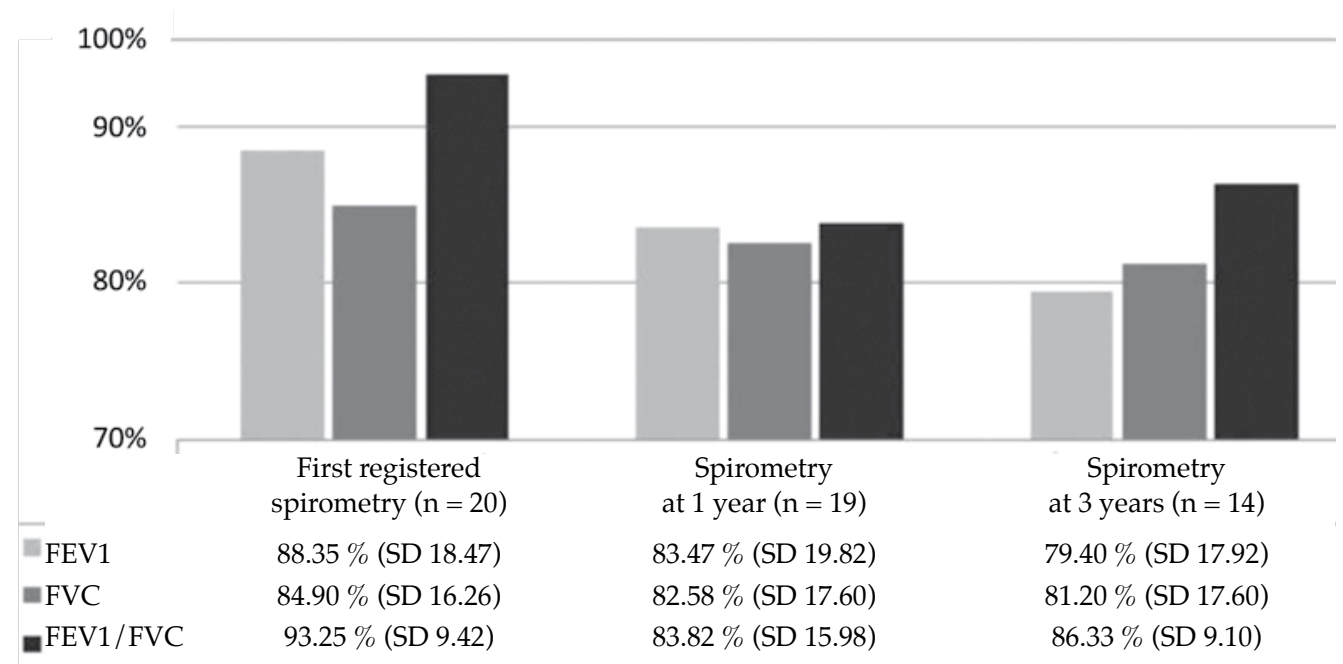

Mean values for forced vital capacity $(\mathrm{FVC})$, forced expiratory volume in the first second $\left(\mathrm{FEV}_{1}\right)$, and $\mathrm{FEV}_{1} / \mathrm{FVC}$ ratio in the spirometries done at baseline, 1 year, and 3 years.

SD: standard deviation. 
In our study, a large percentage of patients developed gastrointestinal and respiratory complications over the follow-up period. The most common complication overall was gastroesophageal reflux, which had been described before. ${ }^{9}$ Two-thirds of patients required anti-reflux treatment. The second most common complication was respiratory tract infection. Exacerbations were observed in almost half of patients; pneumonia, in $27.4 \%$; and conditions related to aspiration syndrome, in up to $5 \%$. It is worth noting that $21.4 \%$ of patients had at least 2 pneumonia events; such high percentage of recurrent pneumonia had been described in other studies ${ }^{10}$ and underlined the importance of diagnosis and an early, aggressive management of these patients in case of a respiratory exacerbation. Additionally, our study found a relation between gastroesophageal and respiratory disease, and patients diagnosed with gastroesophageal reflux had a greater risk for wheezing, exacerbations, and pneumonia, which supported the need for a multidisciplinary approach in these patients.

More than one-third of patients had an abnormal lung function pattern in the first spirometry; over time, the percentage of patients with alterations in lung function was even higher. Therefore, respiratory tract abnormalities and early complications conditioned the course of lung function in these patients, which frequently led to abnormal spirometry patterns. ${ }^{4}$

Some mechanisms that accounted for such variability in lung function patterns were rib cage deformities secondary to surgery, bronchial hyperresponsiveness, persistent inflammation, and tracheomalacia. ${ }^{6}$

In our cohort of patients, the restrictive pattern was more common than the obstructive and mixed patterns. Disparity was observed in the outcomes of previous studies, with some describing a higher prevalence of restrictive patterns ${ }^{5}$ and others showing a predominant obstructive pattern. ${ }^{6,10}$

However, in our study, given the low percentage of patients with available data, conclusions should be made cautiously because, most likely, patients in whom lung function was expected to be mostly altered were precisely those in whom tests could not be performed adequately.

Both FVC and $\mathrm{FEV}_{1}$ reduced over time during the follow-up of these patients, which apparently translated into a progressive worsening since the first years of life. In this regard, and although the sample of patients with available lung function data in the pediatric age was small, our outcomes were consistent with those previously published, which indicated that lung function deterioration and abnormal patterns started in the first years of life. Although published data about lung function in pediatric patients with esophageal atresia was limited to studies with few patients, ${ }^{10-12}$ a higher prevalence of asthma, allergies, infectious complications and a worse quality of life related to long-term respiratory problems had been described in adults. ${ }^{13}$ Notwithstanding this, it was striking that only less than a quarter of patients received follow-up by the pediatric pulmonologist of our hospital, and it was not possible to establish precisely if this was due to the absence of symptoms in the first years or because they continued with follow-up at their referral hospital after the surgery. This situation, together with the high prevalence of complications in the first years of life, should call for a consideration about the need for a multidisciplinary approach to this disease since its onset. ${ }^{14,15}$

Some of the limitations of this study are that it was done in a single site and that data were collected retrospectively, which may have resulted in various biases that could have affected the study conclusions. Besides, as mentioned above, among the studied esophageal atresia cases, lung function data were available only for $25 \%$ of patients, which hindered the possibility of drawing significant conclusions. This is probably due to the low rate of follow-up and the difficulty of performing a spirometry in patients who, in general, have multiple diseases.

In relation to the strengths of this study, it is worth noting the large sample of patients, one of the largest series observed in the bibliography on this topic, and the collection of epidemiological, perinatal, and subsequent Pediatric Pulmonology follow-up data from the medical records.

Survival of these patients has improved in recent decades, although their quality of life is greatly affected due to respiratory and gastrointestinal morbidity; therefore, further prospective studies are required to implement a multidisciplinary follow-up protocol that will allow to detect early complications and prevent lung function worsening.

\section{CONCLUSIONS}

In our sample, a relation was established between gastroesophageal and respiratory 
conditions, and patients diagnosed with gastroesophageal reflux showed a higher risk for wheezing, exacerbations, and pneumonia. Likewise, based on lung function tests, the restrictive pattern was more common than the obstructive and mixed patterns. Both FVC and $\mathrm{FEV}_{1}$ reduced over time during the follow-up of these patients, which apparently translated into a progressive worsening since the first years of life.

\section{REFERENCES}

1. Pedersen RN, Calzolari E, Husby S, Garne E. Oesophageal atresia: prevalence, prenatal diagnosis and associated anomalies in 23 European regions. Arch Dis Child. 2012; 97(3):227-32.

2. Kovesi T, Rubin S. Long-term complications of congenital esophageal atresia and / or tracheoesophageal fistula. Chest. 2004; 126(3):915-25.

3. Shaw-Smith, C. Oesophageal atresia, tracheo-oesophageal fistula, and the VACTERL association: Review of genetics and epidemiology. J Med Genet. 2006; 43(7):545-54.

4. Patria MF, Ghislanzoni S, Macchini F, Lelii M, et al. Respiratory Morbidity in Children with Repaired Congenital Esophageal Atresia with or without Tracheoesophageal Fistula. Int J Environ Res Public Health. 2017; 14(10):E1136.

5. Olbers J, Gatzinsky V, Jönsson L, Friberg LG, et al. Physiological studies at 7 years of age in children born with esophageal atresia. Eur J Pediatr Surg. 2015; 25(5):397-404.
6. Dittrich R, Stock P, Rothe K, Degenhardt P. Pulmonary outcome of esophageal atresia patients and its potential causes in early childhood. J Pediatr Surg. 2017; 52(8):1255-9.

7. Ladd WE. The surgical treatment of esophageal atresia and tracheoesophageal fistulas. NEnglJMed.1944;230(21):625-37.

8. Deurloo JA, Ekkelkamp S, Schoorl M, Heij HA, et al. Esophageal atresia: historical evolution of management and results in 371 patients. Ann Thorac Surg. 2002; 73(1):267-72.

9. Tovar JA, Fragoso AC. Gastroesophageal reflux after repair of esophageal atresia. Eur J Pediatr Surg. 2013; 23(3):175-81.

10. Pedersen RN, Markøw S, Kruse-Andersen S, Qvist N, et al. Long-term pulmonary function in esophageal atresia-A case-control study. PediatrPulmonol. 2017; 52(1):98-106.

11. Agrawal L, Beardsmore CS, MacFadyen UM. Respiratory function in childhood following repair of oesophageal atresia and tracheoesophageal fistula. Arch Dis Child. 1999; 81(5):404-8.

12. Beucher J, Wagnon J, Daniel V, Habonimana E, et al. Longterm evaluation of respiratory status after esophageal atresia repair. PediatrPulmonol. 2013; 48(2):188-94.

13. Sistonen S, Malmberg P, Malmström K, Haahtela T, et al. Repaired oesophageal atresia: respiratory morbidity and pulmonary function in adults. EurRespir J. 2010; 36(5):1106-12.

14. DeBoer EM, Prager JD, Ruiz AG, Jensen EL, et al. Multidisciplinary care of children with repaired esophageal atresia and tracheoesophageal fistula.PediatrPulmonol.2016; 51(6):576-81.

15. Thakkar HS, Hewitt R, Cross K, Hannon E, et al. Themultidisciplinary management of complex congenital and acquired tracheo-oesophageal fistulae. PediatrSurg Int. 2019; 35(1):97-105. 